\title{
Tunneling into one-dimensional Josephson chains and Luttinger liquids
}

\author{
Rosario Fazio $^{\text {a,b }}$, Karl-Heinz Wagenblast ${ }^{\text {a }}$ \\ Carsten Winkelholz ${ }^{a}$, and Gerd Schön ${ }^{a}$ \\ a Institut für Theoretische Festkörperphysik, Universität Karlsruhe, D-76128 \\ Karlsruhe, Germany \\ ${ }^{\mathrm{b}}$ Istituto di Fisica, Università di Catania, viale A. Doria 6, 95129 Catania, Italy
}

\begin{abstract}
One-dimensional arrays of Josephson junctions have been studied because of their unusual zero-temperature superconductor-insulator phase transitions. Furthermore, their low-energy Hamiltonian coincides with that of a Luttinger liquid. Hence 1-D Josephson junction arrays provide a physical realization of this model system. In order to explore these similarities we discuss 1-D arrays with defects, making contact to recent studies of transport in Luttinger liquids. We then study Andreev tunneling between a Josephson chain and a normal metal and between a superconductor and a Luttinger liquid. In both cases the current-voltage characteristics strongly deviate from a linear dependence.
\end{abstract}

\section{Introduction}

In recent years Josephson junction arrays (JJA) built from small tunnel junctions have attracted a growing interest $\Gamma$ both from the experimental and the theoretical side [12]. The transport properties of these low-dimensional structures are strongly influenced by interaction effects. Most of the physics associated with charging effects and Coulomb blockade is described by the so-called capacitance model. When the geometrical capacitance of the islands is of the order of picofarads the associated Coulomb energy may exceed the Josephson energy. In this case quantum fluctuations destroy the coherence and the array is insulating at zero temperature. The phase diagram of the superconductorinsulator transition depends the ratio of Josephson coupling and charging energy and on the dimensionality of the system. A one-dimensional Josephson chain undergoes a $T=0$ transition of the Kosterlitz-Thouless-Berezinskii (KTB) type Tas shown by Bradley and Doniach [3]. DissipationTwhich may

Preprint submitted to Elsevier Science 8 January 1996 
be caused by Ohmic shunts $\mathrm{g}$ gives rise to additional structure in the phase diagram [4-7].

The interest in 1-D systems goes further. Interacting 1-D systems are prototypes of non-Fermi liquids Tand have been studied for several years theoretically [8-10] as well as experimentally [11I12]. One-dimensional interacting systems can be described in terms of the Luttinger liquid (LL) model [8]. The low energy excitations of the interacting electron gas in one dimension are long-wavelength spin and charge oscillationsTrather than fermionic quasiparticle excitations. Accordingly the transport properties cannot be described in terms of the conventional Fermi-liquid approach. The density of states shows a power-law asymptotics at low energies. Depending on the sign of the interaction in a quantum wire an arbitrarily weak barrier leads to perfectly reflecting (for repulsive interactions) or transmitting behavior at low voltages [9]. Some experimental evidence for LL behavior has been found in the power-law energy dependence of the single-particle density of states in the Bechgaard salts [11]. The dispersion of spin and charge excitations in quantum wires has also been measured [12].

In this article we point out that a Josephson junction chain is a physical realization of a LL. We first explore the similarities by discussing a Josephson chain with a defect and show that the problem is similar to that discussed for a LL [9]. We then study Andreev reflection between a superconductor and a quantum wire which are coupled via a tunnel barrierTand between a Josephson chain and a normal island.

\section{The model for a Josephson chain}

The model Hamiltonian for a Josephson junction chain contains the electrostatic interaction and the Josephson coupling

$$
H_{\text {chain }}=\frac{1}{2} \sum_{i j} Q_{i} C_{i j}^{-1} Q_{j}-E_{J} \sum_{i} \cos \left(\phi_{i}-\phi_{i+1}\right)
$$

where $Q_{i}$ is the charge and $\phi_{i}$ the phase of the condensate wave function on the island $i$. The charge and phase on equal sites do not commutel[ $\left.\phi_{j}, Q_{i}\right]=2 e i \delta_{i j}$. The interaction is described by the capacitance matrix $C_{i j}$ Twhich is built by the nearest neighbor capacitance $C$ and the ground capacitance $C_{0}$. In general

the electrostatic interaction has a finite range with a screening length (in units of the lattice spacing) of the order of $\lambda=\sqrt{C / C_{0}}$. 
In this article we will restrict ourselves to the case of short range interactions $\Gamma$ $\sqrt{C / C_{0}} \ll 1$ Twhere a typical charging energy is defined by $E_{0}=4 e^{2} / C_{0}$. This model at $T=0$ can be mapped onto a classical two-dimensional XY model [3] $\Gamma$ which undergoes a phase transition of the KTB type at a critical value of the coupling constants $E_{J} / E_{0} \approx 4 / \pi^{2}$. For large values of the Josephson energy the system is superconducting $\Gamma$ while in the opposite limit it is insulating.

In the limit of large $E_{J}$ and low temperatures the system exhibits phase coherence. The low lying excitations can then be expressed in terms of the fluctuations of the phase within the harmonic approximation. The action related to the Hamiltonian (1) expressed by modes with wavevector $k$ and frequency $\omega_{n}$ is

$$
\mathcal{A}=\frac{1}{2} \sum_{k, \omega_{n}}\left\{C(k) \hbar^{2} \omega_{n}^{2} / 4 e^{2}+E_{J} k^{2}\right\}\left|\phi_{k, \omega_{n}}\right|^{2} .
$$

Here $\omega_{n}=2 \pi n / \hbar \beta$ are the Matsubara bosonic frequencies $\Gamma$ and $C(k)$ is the Fourier transformed of the capacitance matrix. This representation will allow us to establish the connection to the physics of the LL as will become evident in the following.

\section{Josephson chain with a defect}

In this first section we discuss a Josephson chain with a weak link in the middle. The system bears similarities to a LL with a barrier Twhich was discussed by Kane and Fisher [9]. It is convenient to describe the system in the path integral formalism. The action is given by

$$
\mathcal{A}=\sum_{i} \int_{0}^{\beta} d \tau\left[\frac{E_{0}}{2}\left(\frac{d \phi_{i}}{d \tau}\right)^{2}-E_{J}^{(i)} \cos \left(\phi_{i+1}-\phi_{i}\right)\right] .
$$

The notation is the same as in Eq. (1) Texcept for the site-dependent Josephson coupling. A weak link in the chain at site 0 is described by $E_{J}^{(0)} \ll E_{J}$ (while $E_{J}^{(i)}=E_{J}$ for $i \neq 0$ ). If $E_{J} \gg E_{0}$ it is possible to use the harmonic approximation for the junctions of the chain Texcept for the one at $i=0$. Following the approach of Ref. [9] we derive in this way an effective action for the weak junction

$$
\mathcal{A}_{\text {eff }}=\frac{1}{2} g \int \frac{d \omega}{2 \pi}|\omega||\phi(\omega)|^{2}-E_{J}^{0} \int_{0}^{\beta} d \tau \cos (\phi) .
$$


The interaction parameter is given by

$$
g=\alpha \sqrt{\frac{E_{J}}{E_{0}}},
$$

where $\alpha$ is equal to one in the harmonic approximation for the chain. Its exact value can be determined by Monte Carlo simulations (see below). The effective action (4) is identical to the one obtained for a dissipative particle in a periodic potential [18] and also for a spinless LL with a barrier [9].

The harmonic approximation is valid when $g>1$ Ttherefore the present model maps onto the attractive case described in Ref. [9]. In this case ${ }^{2}$ in the renormalization group flow $\Gamma E_{J}^{0}$ flows to larger values. This means that at large distances the effect of the weak link decaysTand the properties of our system reduce to those of a chain with all Josephson coupling equal to $E_{J}$.

We have carried out Monte Carlo simulations of a Josephson chain with one defect using the techniques of Refs. [19]. This enables us to study the validity of the approximations and the renormalization of the weak link in the Josephson chain. The result for the superfluid density Which is a direct measure of the strength of the weak link Tis shown in Fig. 1. The data show the renormalization of the weak link according to the scaling of Ref. [9] Гi.e. $\rho_{0}(L) \sim L^{(1-1 / g)}$. The scaling is cut off by the finite superfluid density of the bulk. We obtain the best fit for $\alpha=1.56$ Tindependent of the strength of the defect and the bulk coupling. This renormalization of $\alpha$ compared to the value $\alpha=1$ in the harmonic approximation is due to phase slips Twhich also drive the superconductor to insulator transition. The value of $\alpha$ implies that $g=1$ roughly coincides with the critical ratio of $E_{J} / E_{0}$ where the KTB transition of the uniform chain has been found. It still remains an open question whether the two values actually coincide rigorously.

The physics of the LL with attractive interaction $g>1$ can be studied in Josephson chains with $E_{J} / E_{0}>1$ Which make them an interesting Texperimentally accessible system for studying models with non-Fermi liquid behavior. On the other hand 5 the LL model with $g<1$ cannot be realized in a Josephson chain. The reason is that in the $E_{J} / E_{0}<1$ (which would correspond to $g<1$ ) the chain is insulating and the low-lying excitations are no longer spin-waves with an acoustic branch. As a consequence the effective action (4) does not contain an Ohmic kernel as required to describe LLs.

\section{Andreev current into a Josephson chain}

In this section we study Andreev tunneling between a normal metal and a Josephson chain [13]. Quantum fluctuations of the phases in the JJA produce 
a nonlinear Andreev current Twhich is very sensitive to the critical properties of the JJA. The Hamiltonian of the system under consideration is

$$
H=\sum_{\sigma} \int_{N} d^{3} r \psi_{\sigma}^{\dagger}(\vec{r}) \epsilon(-i \nabla) \psi_{\sigma}(\vec{r})+T\left[\mathrm{e}^{i \phi_{0}} \psi_{\uparrow}(0) \psi_{\downarrow}(0)+\text { h.c. }\right]+H_{\text {chain }} \cdot(5)
$$

The tunneling Hamiltonian is chosen to describe Andreev processes between the normal metal and the first superconducting island $i=0$. The creation operators $\psi_{\sigma}^{\dagger}$ refer to electrons of the normal metal particle and $\epsilon(-i \nabla)=$ $-\frac{\nabla^{2}}{2 m}-\mu$ is the kinetic energy

When a voltage $V$ is applied Tthe Andreev current across the NS boundary is calculated from the time variation of the electron charge on the metal island

$$
I_{N S}=4 e|T|^{2} \int_{-\infty}^{\infty} d t \mathrm{e}^{-2 i e V t} i \Theta(t)\left\langle\left[A^{\dagger}(t), A(0)\right]\right\rangle
$$

where $A(t)=\mathrm{e}^{i \phi_{0}(t)} \psi_{\uparrow}(0, t) \psi_{\downarrow}(0, t)$. This expression demonstrates that key features are contained in the phase-phase correlation function of the superconducting island which is in contact with the normal metal $\Gamma$

$$
g(\tau)=\left\langle\mathcal{T}_{\tau} \mathrm{e}^{i \phi_{0}(\tau)} \mathrm{e}^{-i \phi_{0}(0)}\right\rangle
$$

In the limit $E_{J} \gg E_{0}$ the harmonic approximation (2) can be used $\Gamma$ which amounts to neglecting quantum phase slips. The system possesses quasi-long range order $\Gamma$ i.e. for $\tau \rightarrow \infty$ the correlations decay with a power-law $g(\tau) \sim \mid$ $\left.\omega_{p} \tau\right|^{1-\eta}$ Twith $\omega_{p}=\sqrt{E_{J} E_{0}}$ and $\eta=1+(2 \pi)^{-1} \sqrt{E_{0} / E_{J}}$. As a result the $I-V$ characteristic at low voltage has a power-law behavior

$$
I_{A}=G_{A} \frac{\mathrm{e}^{-\gamma(\eta-1)}}{\Gamma(1+\eta)} \frac{\omega_{p}}{2 e}\left(\frac{2 e|V|}{\omega_{p}}\right)^{\eta} ; \quad e V \ll \omega_{p}
$$

where $\gamma$ is the Euler number. In the classical limit $\eta \rightarrow 1$ the ground state of the chain has the phases aligned and no collective modes are excited. Then the chain behaves as a bulk superconductor with a linear Andreev $I-V$ characteristic.

When $E_{0} / E_{J}$ increases phase-wave collective modes become active. In particular they are excited by the Andreev tunneling events themselves Tand their back-action leads to the anomalous nonlinear characteristics of Eq. (7). The excitations of the chain act like bosonic modes of an electromagnetic environment coupled to the tunneling pair. Indeed the result (7) resembles that 
obtained for single electron tunneling in systems coupled to an electromagnetic environment [14].

In the limit $E_{J} \gg E_{0}$ the one-dimensional JJA acts like a line of linear elements with the equivalent impedance

$$
\frac{Z(\omega)}{h / 2 e^{2}}=\frac{\eta-1}{\sqrt{1-\left(\omega / \omega_{p}\right)^{2}}} \Theta\left(\omega_{p}-\omega\right)
$$

(see Ref. [13] for more details). Notice that the low voltage behavior is dominated by the capacitance to the ground $C_{0} \Gamma$ and a small finite $C$ does not change the behavior of Eq. (7).

When quantum phase slips are included the harmonic approximation (2) remains a fixed point Hamiltonian cordingly in Eq. (7) $\eta$ is replaced by a renormalized value $\Gamma$ which deviates from the phase-wave value near the superconductor-insulator transition. For further details see Ref. [15].

\section{$5 \quad$ Andreev tunneling into a Luttinger liquid}

In this section we consider a LL coupled to a superconductor by a tunnel barrier and study the current through the interface [16I17]. The Hamiltonian in this case is given by

$$
H=H_{S}+H_{T}+H_{L}
$$

The superconductor is described by the standard BCS-Hamiltonian $H_{S}$. The tunneling part is

$$
H_{T}=\sum_{s= \pm 1} \int d x \int d r T(x, r) \Psi_{L, s}^{\dagger}(x) \Psi_{S, s}(r)+\text { h.c. }
$$

Finally $H_{L}$ describes the LL. It can be expressed in terms of boson operators

$$
H_{L}=\int \frac{d x}{\pi} \sum_{j} v_{j}\left[\frac{g_{j}}{2}\left(\nabla \phi_{j}\right)^{2}+\frac{2}{g_{j}}\left(\nabla \theta_{j}\right)^{2}\right] .
$$

It is written as a sum of the contributions from spin $(j=\sigma)$ and charge $(j=\rho)$ degrees of freedom. Here $v_{j}=\left(2 / g_{j}\right) v_{F}$ are the renormalized $\Gamma$ interactiondependent Fermi velocities. For repulsive interaction we have $g_{\rho}<2$ Twhile for 
attractive interaction $g_{\rho}>2$. The fields $\theta$ and $\phi$ are canonically conjugated $\Gamma$ $\left[\phi_{s}(x), \nabla \theta_{s^{\prime}}\left(x^{\prime}\right)\right]=(i \pi / 2) \delta\left(x^{\prime}-x\right) \delta_{s, s^{\prime}}$.

The similarity to the Josephson chain becomes manifest if we write the corresponding action for a LL

$$
\mathcal{A}_{L}=\frac{1}{2} \sum_{k, \omega_{n}} \sum_{j}\left\{g_{j} \omega_{n}^{2} / v_{j}+g_{j} v_{j} k^{2}\right\}\left|\phi_{k, \omega_{n} ; j}\right|^{2}
$$

Comparing Eq. (12) and Eq. (2) it is clear that the Josephson chain corresponds to a spinless LL. This offers the possibility to study the interaction effects of a LL by studying a one-dimensional Josephson array.

In the following we will consider spin-independent interactions. Hence $\Gamma g_{\sigma}=2$ and $v_{\sigma}=v_{F}$. For an infinitely extended quantum wire the Fermi field operators can be expressed by boson operators as

$$
\Psi_{s}^{\dagger}=\sqrt{\rho_{0}} \sum_{m= \pm 1} \mathrm{e}^{i k_{F} m x} \mathrm{e}^{i m \theta_{s}(x)} \mathrm{e}^{i \phi_{s}(x)}
$$

where $\theta_{s}=\frac{1}{\sqrt{2}}\left(\theta_{\rho}+s \theta_{\sigma}\right)$ and $\phi_{s}=\frac{1}{\sqrt{2}}\left(\phi_{\rho}+s \phi_{\sigma}\right)$. The density of electrons per spin in the LL is $\rho_{0}=k_{F} / 2 \pi$. For transparency we will assume in the following that the gap of the superconductor is much larger than all other characteristic energies of the system.

We first evaluate the contribution of Andreev tunneling to the nonlinear subgap current. The current is evaluated in standard way in an expansion in the tunneling Hamiltonian

$$
I / e=\left\langle\dot{N}_{L}(t)\right\rangle=\left\langle\bar{T} \mathrm{e}^{i \int_{-\infty}^{t} H_{T_{I}}\left(t^{\prime}\right) d t^{\prime}} \dot{N}_{L_{I}}(t) T \mathrm{e}^{-i \int_{-\infty}^{t} H_{T_{I}}\left(t^{\prime}\right) d t^{\prime}}\right\rangle .
$$

The terms contributing to the current can be classified diagrammatically. The important element is

$$
\Pi_{s, s^{\prime}}\left(x_{i}, t\right)=\left\langle\Psi_{L, s}\left(x_{3}, t_{3}\right) \Psi_{L,-s}\left(x_{2}, t_{2}\right) \Psi_{L, s^{\prime}}^{\dagger}\left(x_{1}, t_{1}\right) \Psi_{L,-s^{\prime}}^{\dagger}(x, t)\right\rangle .
$$

As long as all energies ( $e V \Gamma k T)$ are small compared to the gap of the superconductor the anomalous correlations in the superconductor can be approximated by delta-functions. The expression for the current then reads

$$
I=16 N(0)^{2} \pi^{2}|T|^{4} e \operatorname{Re}\left\{\int_{0}^{\infty} d t \Pi_{+,-}\left(t_{3}=t_{2}=0, t_{1}=t\right)\left(\mathrm{e}^{2 i e V t}-\mathrm{e}^{-2 i e V t}\right)\right\} .
$$


The averages can be evaluated by using Eq. (11). The resulting current at $T=0$ is

$$
\begin{aligned}
I(V, T=0)= & \frac{1}{4} G_{T}^{2} R_{K} V\left(\frac{2 e V}{E_{F}}\right)^{\frac{2}{g_{\rho}}+\frac{g_{\rho}}{2}-2} \frac{1}{\Gamma\left(\frac{g_{\rho}}{2}+\frac{2}{g_{\rho}}\right)}\left(\frac{2}{g_{\rho}}\right)^{\frac{2}{g_{\rho}}+\frac{g_{\rho}}{2}} \\
& +\frac{1}{4} G_{T}^{2} R_{K} V\left(\frac{2 e V}{E_{F}}\right)^{\frac{2}{g_{\rho}}-1} \frac{1}{\Gamma\left(1+\frac{2}{g_{\rho}}\right)}\left(\frac{2}{g_{\rho}}\right)^{\frac{2}{g_{\rho}}}
\end{aligned}
$$

where $G_{T}$ is the dimensionless tunneling conductance. For repulsive electronelectron interaction the term $I \sim V^{2 / g_{\rho}}$ dominates while for attractive electronelectron interaction $I \sim V^{\left(2 / g_{\rho}\right)+\left(g_{\rho} / 2\right)-1}$ is the relevant one. These power laws confirm the qualitative expectation $\Gamma$ a suppression or increase of the current by repulsive or attractive interactions $\Gamma$ respectively. Accordingly the linear conductance $G=\partial I / \partial V \sim V^{\gamma-1}$ (where $\gamma$ is the leading power in Eq. (17)) at $V=0$ and $T=0$ vanishes for repulsive interaction $(\gamma>1)$ Tor becomes infinite for attractive interaction $(\gamma<1)$.

At finite temperatures we have

$$
G=\frac{1}{4} G_{T}^{2} R_{K}\left[c_{1}\left(\frac{k T}{E_{F}}\right)^{\frac{g_{\rho}}{2}+\frac{2}{g_{\rho}}-2}+c_{2}\left(\frac{k T}{E_{F}}\right)^{\frac{1}{g_{\rho}}-1}\right]
$$

where $c_{1}, c_{2}$ are constants of order one.

The divergence of the conductance in the attractive case reveals the failure of the perturbation expansion in the tunneling Hamiltonian. By means of a renormalization group ( $R G$ ) approach it is possible to identify the Cooper pair channel (tunneling of two electrons with opposite spin on different branches) as the relevant process for attractive interactions (in the $\mathrm{RG}$ sense). More generally if $g_{\sigma} \neq 2 \Gamma$ the fixed point is shifted from $g_{\rho}=2 \Gamma$ and in principle one may have a fully transparent barrier for Andreev tunneling even in the presence of repulsive interaction in the LL.

\section{Discussion}

In this paper we discussed the possibility of using one-dimensional Josephson junction arrays to detect transport properties which are currently investigated in Luttinger liquids. A direct evidence of this connection was discussed in the first part of the paper when the Josephson chain with a weak link was considered. In the second part of the paper we investigated Andreev-type transport 
and showed that the $I-V$ characteristics for the Josephson chain and the LL coincide. A key point in the discussion is the observation that in the superconducting chain the low-lying excitations are spin-waves $\Gamma$ which are the equivalent of the boson modes in LLs. The analogy between the two models becomes apparent if we note that the the creation operator for an extra charge in a island is given by $\sim \exp (i \theta)$ Twhich is very similar to the representation (13) of Fermion creation operators. The chain does not possesses any internal degree of freedom and accordingly is mapped onto the spinless LL. In principle one may think to use coupled chain to get also model for spin- $1 / 2$ electron in one dimension.

\section{Acknowledgement}

The work on the Andreev tunneling in a Josephson chain was done in collaboration with Giuseppe Falci and Arturo Tagliacozzo. This work is supported by the DFG within the research program of SFB 195.

\section{References}

[1] see e.g. Proceedings of the NATO ARW Coherence in Superconducting Networks, Eds. J.E.Mooij and G. Schön, Physica B152, (1988); J.E. Mooij and G. Schön in Single Charge Tunneling, Eds. H. Grabert and M.H. Devoret, NATO ASI series Vol. 294 (Plenum Press, New York 1992); Mesoscopic Superconductivity, Eds. F.W.J. Hekking, G. Schön, and D.V. Averin, (Elsevier 1994) and Physica B 203 (1994).

[2] L.J. Geerligs, M. Peters, L.E.M. de Groot, A. Verbruggen and J.E. Mooij, Phys. Rev. Lett 63, 326 (1989); H.S.J. van der Zant, L.J. Geerligs and J.E. Mooij, Europhys. Lett. 19, 541 (1992); S. Doniach, Phys. Rev. B 24, 5063 (1981) R. Fazio and G. Schön, Phys. Rev. B 43, 5307 (1991).

[3] R.M. Bradley and S. Doniach, Phys. Rev. B 30, 1138 (1984).

[4] S.E. Korshunov, Europhys. Lett. 9, 107 (1989).

[5] W. Zwerger, Europhys. Lett. 9, 421 (1989).

[6] P.A. Bobbert, R. Fazio, G. Schön and G.T. Zimanyi, Phys. Rev. B 41, 4009 (1990).

[7] P.A. Bobbert, R. Fazio, G. Schön and A.D. Zaikin, Phys. Rev. B 45, 2294 (1992).

[8] V.J. Emery, in Highly Conducting One-Dimensional Solids, edited by J.T. Devreese, R.P. Evrard, and V.E. van Doren (Plenum Press, New York 1979); J. Sólyom, Adv. Phys. 28, 201 (1979). 
[9] C.L. Kane and M.P.A. Fisher, Phys. Rev. Lett. 68, 1220 (1992); K.A. Matveev and L.I. Glazman, Phys. Rev. Lett. 70, 990 (1993).

[10] R. Fazio, F.W.J. Hekking, and A.A. Odintsov, Phys. Rev. Lett. 74, 1843 (1995).

[11] B. Dardel, D. Malterre, M. Grioni, Y. Baer, J. Voit, and D. Jérôme, Europhys. Lett. 24, 687 (1993).

[12] A.R. Goñi, A. Pinczuk, J.S. Weiner, J.M. Calleja, B.S. Dennis, L.N. Pfeiffer, and K.W. West, Phys. Rev. Lett. 67, 3298 (1991).

[13] G. Falci, R. Fazio, A. Tagliacozzo, and G. Giaquinta, Europhys. Lett. 30, 169 (1995).

[14] G.L. Ingold and Yu.V. Nazarov, in Single Charge Tunneling, Eds. H. Grabert and M.H. Devoret, (Plenum Press, New York 1992) p. 221.

[15] J.V. José, L.P. Kadanoff, S. Kirkpatrick and D.R. Nelson, Phys. Rev. B16, 1217 (1977).

[16] M.P.A. Fisher, Phys. Rev. B 49, 14550 (1994).

[17] C. Winkelholz, R. Fazio, F.W.J. Hekking, and G. Schön, (unpublished).

[18] A. Schmid, Phys. Rev. Lett. 51, 1506 (1983).

[19] E.S. Sørensen, M. Wallin, S.M. Girvin, and A.P. Young, Phys. Rev. Lett. 69, 828 (1992); A. van Otterlo and K.H. Wagenblast, Phys. Rev. Lett. 72, 3598 (1994); A. van Otterlo and K.H. Wagenblast, R. Baltin, C. Bruder, R. Fazio, and G. Schön, to appear in Phys. Rev. B 52 (1995). 


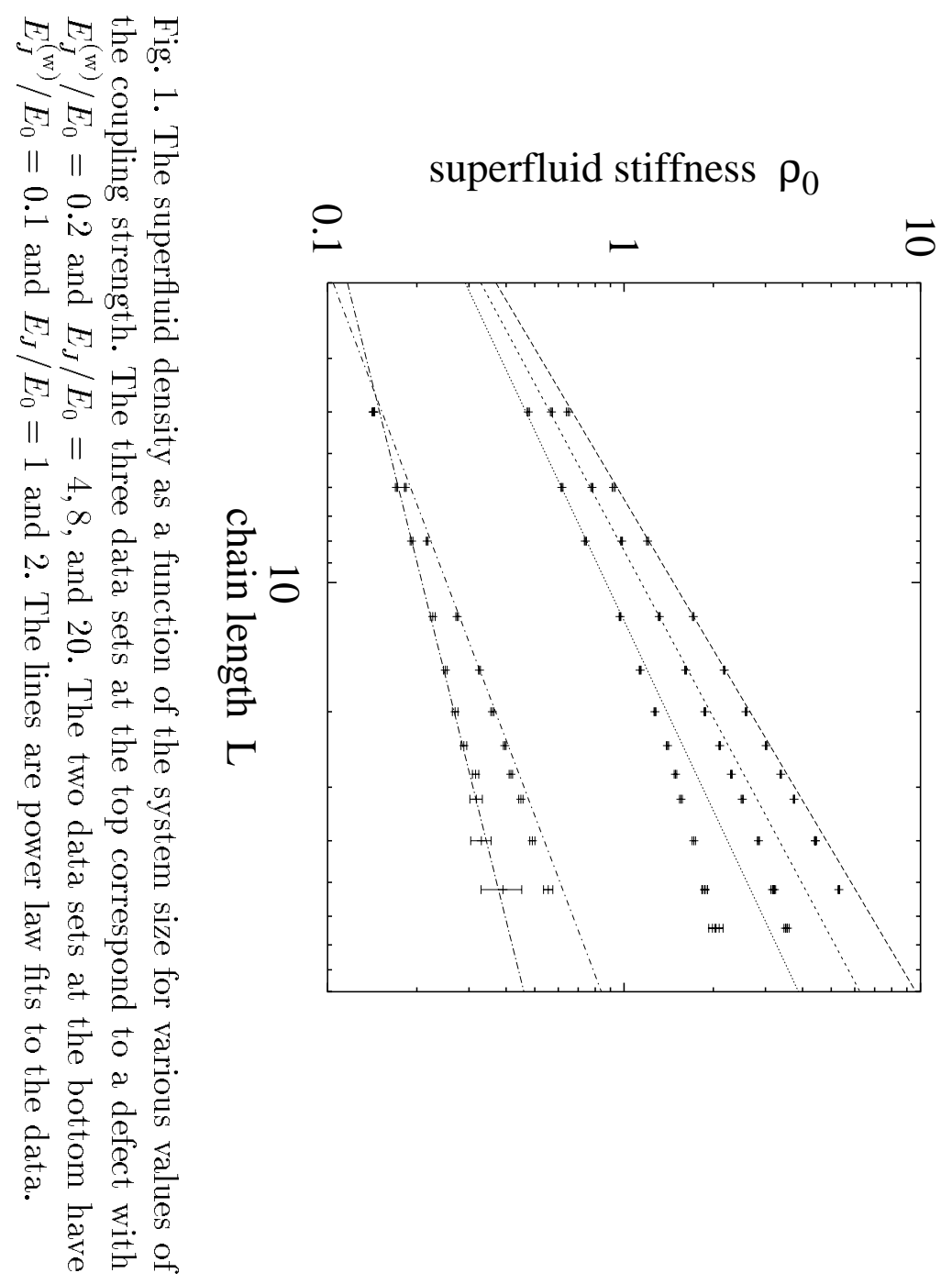

